\title{
DEGRADATION OF FOOD MANUFACTURING WASTES BY A FUNGAL ISOLATE
}

\author{
Felipe Gordillo ${ }^{1 *}$, Oscar Candia ${ }^{2}$, Alex R. Gonzalez ${ }^{3}$, Paulina Pradel ${ }^{4}$, Cecilia Cordero², Rodrigo \\ Diaz $^{2}$, Rolando Garcia-Gonzalez ${ }^{1}$
}

${ }^{1}$ Centro de Biotecnología de los Recursos Naturales, Facultad de Agronomía y Ciencias Forestales, Universidad Católica del Maule, Avda. San Miguel 3605, Talca, Chile.

${ }^{2}$ Centro de Estudios de Alimentos Procesados - CEAP, Talca, Chile.

${ }^{3}$ Laboratorio de Microbiología de Extremófilos, Departamento de Ciencias Biológicas, Universidad de los Lagos, Avda. Alcalde Gustavo Fuchslocher 1305, Osorno, Chile.

${ }^{4}$ Laboratorio de Fisiología y Biología Molecular Vegetal, Departamento de Ciencias Agronómicas y Recursos Naturales, Universidad de la Frontera, Avda. Francisco Salazar 01145, Temuco, Chile..

*Corresponding author E-mail: fgordillo@ucm.cl

\begin{abstract}
Food manufacturing wastes, such as olive mill solid waste, tomato pomace and grape pomace are three wastes produced by food industries in the Region of Maule. One of the simplest strategies for the proper disposal of these residues is to directly eliminate them by cultivation with degrading mesophilic microorganisms, which use these wastes as their sole carbon source. A fungal strain was isolated from tomato peel and used to degrade food manufacturing wastes. This strain was effective in degrading olive mill solid waste, but partially degraded both grape and tomato pomace. Based on mycelium morphology, sequence of part of the ribosomal and internal transcribed spacer (ITS) DNA, the strain most probably belongs to Botrytis genera. This is the first report that uses a strain similar to Botrytis in degrading food manufacturing wastes from the Region of Maule.
\end{abstract}

Key words: food manufacturing wastes, Botrytis, olive mill solid waste, tomato pomace, grape pomace.

\section{INTRODUCTION}

The release of contaminants into the environment is one of the main causes of damage to the biosphere. Most of these compounds are introduced gradually due to the increase in human activities, such as agriculture, food industry and mining. These anthropologic forces pose a threat to the health of flora, fauna and humans through the disposal of waste waters, soil contaminants, and release of toxic gasses into the atmosphere (Checa-Vizcaino et al., 2016). At present, special efforts are being made to develop new methods and technologies that can be applied to contaminated soil, water and air in order to reduce negative ecological and environmental effects resulting from human activities (Khan et al., 2004; Onwubuya et al., 2009; Desai et al., 2010; Gill et al., 2014).
Olive-mill solid waste (OMSW), tomato pomace (TP) and grape pomace (GP) are three important food manufacturing wastes (FMWs) from the Region of Maule, Chile. OMSW is a by-product waste produced from olive oil elaboration by a two-phase centrifugation system. The chemical characterization of OMSW has been determined, showing that it has a high moisture content, a slightly acidic $\mathrm{pH}$ and a high content of organic matter, mainly composed of lignin, hemicelluloses and cellulose (the so-called lignocellulose complex); it also presents a high fat content and hydrosolubles polyphenols. Due to its antimicrobial and phytotoxic properties, OMSW has been described as a severe contaminant for soil and rivers if not properly disposed (Alburquerque et al., 2004; Rubio-Senent et al., 2013). On the other hand, TP is a by-product of tomato mainly consisting of peels and seeds, which is rich in fiber, 
sugars, proteins, pectins, minerals and fats (Del Valle et al., 2006), while GP is mainly composed of pectins, glucans, alcohol, phenols and flavonoids (Baydar et al., 2007; Deng et al., 2011; Fontana et al., 2013).

As above mentioned, FMWs mainly consist of a mixture of polymers, which are composed of cellulose, lignin, pectin and hemicellulose. Cellulose is a homopolimer composed of glucose units; lignin is a polyphenol whose main monomer is p-hidroxiphenylpropane; and pectin is a polymer comprised of $\beta$-D-galacturonic acid. Finally, hemicellulose corresponds to the fraction from lignocellulose, which is removed by alkali and is composed by a mixture of complex heteropolymers of xylan, galactan, mannan and arabinan. The strength of the cell wall is established by the presence of several types of interactions between these polymers. In this way, hemicellulose interacts with cellulose by means of hydrogen bonds, while lignin interacts by means of eter or ester covalent bonds (Pérez et al., 2002).

In nature, a wide range of fungal strains are capable of using FMWs as sole carbon source. These fungal strains secrete a wide range of enzymes with hydrolytic potential that can degrade complex polymers of sugars, such as those present in the cell wall (Sanchez, 2009). Fungal enzymes from Penicillium purpurogenum, Neurospora crassa and Mucor sp. have been used in the degradation of coffee silverskin and spent coffee grounds generated by the coffee industry, resulting in a higher release of phenolic compounds that can be applied either in the pharmaceutical or food industries (Machado et al., 2012). Pleurotus ostreatus has been used to degrade TP for the production of laccase enzyme (Freixo et al., 2012). In addition, the same substrate has been used to produce xylanase by Aspergillus awamori (UmszaGuez et al., 2011).

The objective of this work was to isolate a fungal strain capable of growing on food manufacturing wastes (FMW) in order to generate future strategies for the disposal of FMWs in the Region of Maule, Chile.

\section{MATERIALS AND METHODS}

Chemicals. From Merck (Darmstadt, Germany): $\left(\mathrm{NH}_{4}\right)_{2} \mathrm{SO}_{4}, \mathrm{KH}_{2} \mathrm{PO}_{4}, \mathrm{MgSO}_{4}, \mathrm{FeSO}_{4}$ x $7 \mathrm{H}_{2} \mathrm{O}$, isopropanol, chloroform. From Phytotechnology Laboratories (Kansas, USA): $\mathrm{CaCl}_{2^{\prime}} \mathrm{ZnSO}_{4^{\prime}} \mathrm{CoCl}_{2^{\prime}}$ $\mathrm{MnSO}_{4}$. From Promega (Madison, USA): Urea. From Becton, Dickinson and Company: Neopeptone, Potato Dextrose Agar. From Sigma-Aldrich (Missouri, USA): Gallic acid, Folin-Ciocalteu reagent and glucose.
Fungal strain and culture conditions. A fungal strain was isolated from tomato and kept refrigerated at $4^{\circ} \mathrm{C}$ for at least one week. Tomato skins were cut into small pieces, placed on the surface of a potato dextrose agar plate (PDA) and kept at $28^{\circ} \mathrm{C}$ in an incubator. After seven days, small pieces of agar containing fungi were extracted from the edge of the mycelium and placed on the center of a new PDA plate. Plates were incubated for 7 days at $28^{\circ} \mathrm{C}$. This procedure was repeated two more times until homogeneous colony formation. After the agar plate was fully covered with mycelium, dark sclerotia were formed. The fungal slide culture procedure was used for mycelium staining. Briefly, a plug of a PDA plate of $0.4 \mathrm{~mm}^{2}$ was placed on a glass slide. Mycelium was placed on the plug and covered with a microscope coverslip. After mycelial growth on the PDA plug and attachment on the coverslip occurred, mycelia was carefully removed and stained with a solution of lactophenol cotton blue, and then observed using an optical microscope (Optika, Italy). Liquid cultures were performed in Mandel's medium as previously described (Ravanal et al., 2013). Four agar plates containing the mycelium were scraped using an inoculation loop. The mycelium was dissolved in an Erlenmeyer flask containing a sterile $0.9 \% \mathrm{NaCl}$ solution and glass beads to facilitate spore release. After shaking, this solution was filtered using sterile dressing. Spores were counted in a Petroff-Hauser chamber and $4 \times 10^{6}$ were inoculated in $100 \mathrm{~mL}$ of Mandel's medium supplemented with FMWs at $0.5 \%$ as the only carbon source. For solid medium experiments, $0.6 \mathrm{~mm}^{2}$ of a PDA plug containing the mycelium were extracted and placed on the surface of the plates containing Mandel's medium containing $4 \%$ of agar (Difco) and the FMWs at $0.5 \%$.

Strain identification. Mycelium was resuspended in $1 \mathrm{~mL}$ of modified Buffer $\mathrm{A}$ and then lysed in Precellys ${ }^{\circledR} 24$ (Bertin, France) using beads of $0.5 \mathrm{~mm}$. After lysis, tubes were centrifuged at $11,000 \mathrm{rpm}$ for $10 \mathrm{~min}$ at $4^{\circ} \mathrm{C}$. Then the supernatant was transferred to a clean tube and an equal volume of isopropanol was added. Tubes were kept at $-20^{\circ} \mathrm{C}$ overnight. Samples were centrifuged at $12,000 \mathrm{rpm}$ for $10 \mathrm{~min}$ at $4^{\circ} \mathrm{C}$ and the supernatant was discarded. Samples were dried at room temperature and then resuspended in Milli Q water. DNA was purified with 1 volume of phenol/chloroform and centrifuged at 11,000 $\mathrm{rpm}$ for $5 \mathrm{~min}$ at $4^{\circ} \mathrm{C}$. Then, 2.5 volumes of cold ethanol were added and then kept overnight at $-20^{\circ} \mathrm{C}$. DNA was washed with ethanol $70 \%$ and dried at room temperature. DNA was resuspended in MQ water and then stored at $-20^{\circ} \mathrm{C}$. Finally, it was quantified using Nanodrop 2000 (Thermo 
Scientific, Delaware, USA) and visualized using agarose $1 \%$ gel electrophoresis. Aliquots were used in PCR reactions using primers ITS-1 $\left(5^{\prime}\right.$ TCC GTAGGTGAACCTGCGG 3') and ITS-4 (5' TCCTCCGCTTATTGATATGC 3'). An aliquot of this PCR reaction was sequenced using the same primers (Macrogen, Korea)(Cáceres et al., 2012).

The obtained sequence was compared with all sequences from GenBank using the BLAST alghoritm (Megablast) (http://blast.ncbi.nlm.nih. gov/Blast.cgi). Bioedit software was used for multiple alignments and edition of selected sequences. Phylogenetic analyses were carried out using Mega 7. The phylogenetic tree was constructed using the Neighbor-joining method. This method was used to infer the evolutionary history of the isolates, while the bootstrap consensus tree was inferred from 100 replicates. There were 405 and 184 positions in the final dataset. Botrytis genera were used as the outgroup (Kumar et al., 2016).

Residue preparation. FMWs were dried at $60^{\circ} \mathrm{C}$ in a hot plate for at least 24 hours avoiding burning. Subsequently, they were ground in a crushing machine and sieved in a $300 \mathrm{~mm}$ mesh (VWR, Pennsylvania, USA). This material was used at $0.5 \%$ as the carbon source for liquid and solid medium experiments (Sanchez C., 2009).

Analytical methods. Mycelium radial growth was monitored by measuring the colony diameter every 24 hours. The total content of phenolic compounds in the supernatant of the culture was measured using the Folin-Ciocalteu reagent, using gallic acid as standard. Total carbohydrates were measured using the Dubois method using glucose as standard (Valenzuela et al., 2015). A volume of $500 \mu \mathrm{L}$ of culture supernatants was used in both methods. However, a volume of 500 $\mu \mathrm{L}$ of $1 / 10$ dilution was used for total sugar analysis in the case of Mandel's medium supplemented with glucose.

\section{RESULTS AND DISCUSSION}

In order to evaluate the degradation of FMWs, a fungal strain was isolated from decomposed tomato kept in the refrigerator. The fungal strain was grown in PDA plates for seven days, until it completely covered the Petri plate. Black sclerotic bodies were observed 9-10 days after (Fig. 1A). Microscope observations of mycelium samples stained with lactophenol cotton blue showed the formation of branching tree-like conidiophores and ovoidal conidia. Besides, the hyphae was of a segmented type (Fig. 1B). Inoculation of fungi on raspberry, grape wine and spinach leaves showed dark rings after 5 days of inoculation (data not shown). PCR amplification using ITS-1 and ITS-4 primers using the DNA of the mycelium grown on LB media produced a DNA amplification fragment of around $570 \mathrm{pb}$. This fragment was sequenced using the ITS primers. Partial DNA sequence using ITS- 1 showed a sequence similar to $(100 \%$ identity) Botrytis cinerea strain ATCC 11542 (Genbank: KU729081.1). The analysis of partial sequence of strain T1 showed that this microorganism is closely related to phytopathogens as Botrytis and Alternaria genera (Fig. 1C). Both genera are widely recognized as a cause of severe losses in agriculture. This identification agrees with the morphology of mycelia and the pathogenic behavior of this kind of strains on different biotic surfaces (see above).

After DNA characterization, strain T1 was grown on Mandel's medium supplemented with FMWs. Glucose was added to the liquid cultures and plates for comparison purposes. On plates, strain T1 was capable of growing on the three FMWs. However, the maximum radius at 96 hours was observed on OMSW $(3.6 \mathrm{~cm})$, followed by GP $(3.2 \mathrm{~cm})$ and TP $(2.8 \mathrm{~cm})$ (Fig. 2A). Cultures were incubated at $24^{\circ} \mathrm{C}$ and the total biomass (wet weight) obtained after nine days of culture was $5.6 \mathrm{~g}$ for OMSW, $1.9 \mathrm{~g}$ for GP, and $1.4 \mathrm{~g}$ for TP. It is important to note that, although OMSW waste has been referred to as having antimicrobial properties, the visual inspection of OMSW cultures after 10 days of inoculation revealed almost complete degradation (data not shown). Conversely, GP and TP did not seem to be rich carbon sources for strain T1. Future research is required to determine if the low growth observed in GP and TP wastes, as compared to that in OMSW, could be due to antifungal properties released during metabolization.

To evaluate the metabolization of total sugars after inoculation with strain T1, total carbohydrate content was analyzed from culture supernatants. The total sugar content reached the highest values in OMSW, which decayed on the first day, keeping constant levels after three days of culture. In the GP culture, total sugars decreased after four days of inoculation reaching similar levels to those observed in OMSW cultures. In contrast, almost no changes were found in TP cultures. In the glucose cultures, 1/10 dilution of supernatants decreased sugar content on the fourth day (Fig. 2B). These results suggest the presence of metabolic activity possibly due to extracellular enzymes produced by strain T1 (Espino et al., 2010; González-Fernández et al., 2014). Similarly, total phenolic compounds were measured after inoculation with strain T1. High values of total phenolic compounds were found in OMSW, which decayed after one day of inoculation and reached constant levels on the 

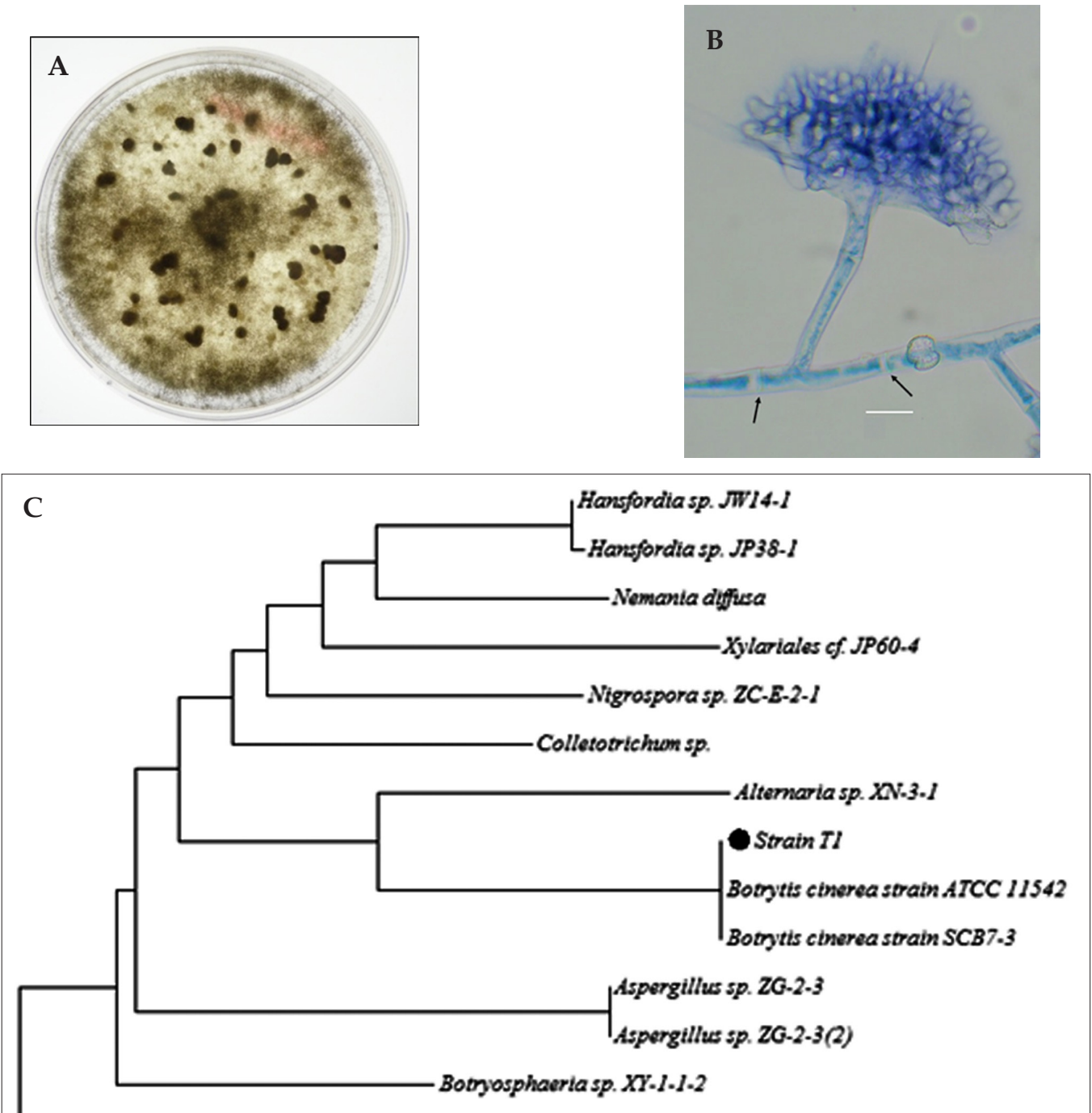

Uncultured Ascomycota clone LH2I

\subsection{5}

Fig. 1. Morphology and molecular analysis of strain T1. A. Mycelium from strain T1 grown on a PDA plate showing dark sclerotia. B. A sample taken from a mycelium grown on PDA plate and stained with lactophenol blue. Sample was observed under $40 \mathrm{X}$ microscope. Bar indicates 20 $\mathrm{mm}$. Dark arrows indicate septa on hypha. Observe the tree-like conidiophores presenting ovoidal conidia. C. Neighbor joining tree of the strain T1 (bold letters), using ITS1 spacer DNA. The results show the relationship of 13 sequences of different fungus genera obtained from GenBank and Botrytis genera as outgroup. The bootstraps value obtained from 100 replicates were shown in each node. Scale bar represents 0.05 substitution per nucleotide position.

fifth day. Similar behavior was found in the GP supernatant, but constant levels were reached on the third day. In contrast, supernatants of TP cultures were almost depleted on phenolic compounds. As expected, residual values were observed in Mandel's medium supplemented with glucose. Those values could be due to the presence of phenolic compounds in Mandel's medium since this contains neopeptone. These results suggest the presence of mechanisms to degrade or transform phenolic compounds from the culture medium present on OMSW and GP cultures (Schouten et al., 2002). It is known that strain T1 secretes laccases that could be responsible for phenolic compounds oxidation (Espino et al., 2010; González-Fernández et al., 2014) (Fig. 2C). These results indicate that 

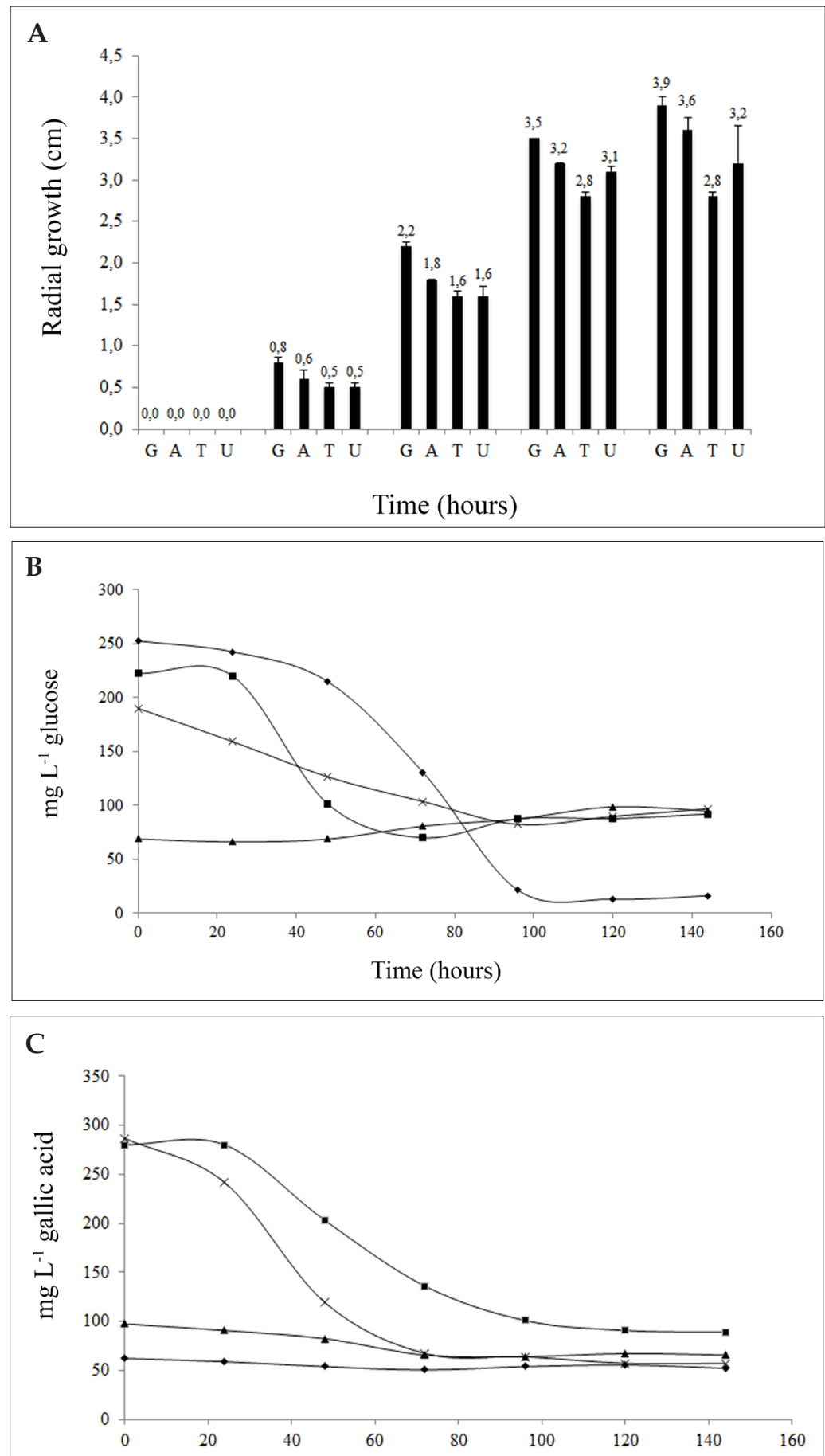

Fig. 2. Growth of strain T1 on food manufacturing wastes. A. Radial growth of strain T1 on agar plates containing Mandel's medium supplemented with glucose (G), OMSW (A), TP (T) and GP (U). Error bars indicate the SDs based on three different replicated experimental values. Numbers on top of each bar indicate the relative radius corresponding to mycelial growth. $B$. Analysis of total sugars from the supernatant of strain T1 grown in different carbon sources. C. Analysis of total phenolic compounds from the culture supernatant of strain T1 grown in different carbon sources. Squares: Mandel's medium supplemented with OMSW. Cross: Mandel's medium supplemented with GP. Triangle: Mandel's medium supplemented with TP. Diamonds: Mandel's medium supplemented with glucose.

OMSW: olive mill solid wastes; TP: tomato pomace; GP: grape pomace. 
mesophilic fungi can be used to degrade FMWs in order to eliminate this kind of waste from the environment.

\section{CONCLUSIONS}

The results show that strain $\mathrm{T} 1$ degrades olive mill solid wastes more efficiently (OMSW) than grape pomace (GP) and tomato pomace (TP). The physiological adaptations of strain T1 when used in food manufacturing wastes (FMW) (i.e. changes in protein expresion), and the adjustment of the parameters of the biodegradation should be studied in order to improve the biodegradation of these three residues, particularly GP and TP wastes, and eventually eliminate them from to environment.

\section{ACKNOWLEDGMENTS}

This work was partially funded by the projects: CONICYT REGIONAL/GORE 326 MAULE/ CEAP/R09I2001; PROJECT 79112042 from PROGRAMA PAI - CONICYT; and PROJECT BIP 3303689-0 from the FONDO DE INNOVACION PARA LA COMPETIVIDAD, REGIÓN DEL MAULE. It was also financially supported by projects DIULA 09/2014 to AG, CONICYT Doctoral Fellowship N 21130177 to PP.

\section{LITERATURE CITED}

Alburquerque, J. 2004. Agrochemical characterisation of "alperujo", a solid by-product of the two-phase centrifugation method for olive oil extraction. Bioresourse Technology 91:195-200.

Baydar, N. G., G. Özkan, and E. S. Çetin. 2007. Characterization of grape seed and pomace oil extracts. Grasas y Aceites 58:29-33.

Cáceres, P., C. Cordero, G. González, K. Quiroz, J. Bobadilla, C. Bravo, P. Caligari, B. Carrasco, and R. García-Gonzales. 2012. Efficient protocols for the extraction of microbial DNA from the rhizosphere of hydrophilic forests in Chile. Ciencia e Investigación Agraria 39:585-592.

Checa-Vizcaíno, M. A., M. González-Comadran, and B. Jacquemin. 2016. Outdoor air pollution and human infertility: a systematic review. Fertility and Sterility 282:62548-62557.

Del Valle, M., M. Camara, and M. E. Torija. 2006. Chemical characterization of tomato pomace. Journal of the Science of Food and Agriculture 86:1232-1236.
Deng, Q., M.H. Penner, and Y. Zhao. 2011. Chemical composition of dietary fiber and polyphenols of five different varieties of wine grape pomace skins. Food Research International 44:2712-2720.

Desai, C., H. Pathak, and D. Madamwa. 2010. Advances in molecular and "-omics" technologies to gauge microbial communities and bioremediation at xenobiotic/anthropogen contaminated sites. Bioresource Technology 101:1558-1569.

Espino, J.J., G. Gutiérrez-Sánchez, N. Brito, P. Shah, R. Orlando, and C. González. 2010. The Botrytis cinerea early secretome. Proteomics 10:3020-3034.

Fontana, A.R., A. Antoniolli, and R. Bottini. 2013. Grape pomace as a sustainable source of bioactive compounds: Extraction, characterization, and biotechnological applications of phenolics. Journal of Agricultural and Food Chemistry 61:8987-9003.

Freixo, M.R., A. Karmali, and J.M. Arteiro. 2012. Production, purification and characterization of laccase from Pleurotus ostreatus grown on tomato pomace. World Journal of Microbiology and Biotechnology 28:245-254.

Gill, R.T., M.J. Harbottle, J.W.N. Smith, and S.F. Thornton. 2014. Electrokinetic-enhanced bioremediation of organic contaminants: a review of processes and environmental applications. Chemosphere 107:31-42.

González-Fernández, R., K. Aloria, J. Valero-Galván, I. Redondo, J.M. Arizmendi, and J.V. Jorrín-Novo. 2014. Proteomic analysis of mycelium and secretome of different Botrytis cinerea wild-type strains. Journal of Proteomics 97:195-221.

Khan, F.I., T. Husain, and R. Hejazi. 2004. An overview and analysis of site remediation technologies. Journal of Environmental Management 71:95-122.

Kumar, S., G. Stecher, and K. Tamura. 2016. MEGA7: Molecular Evolutionary Genetics Analysis Version 7.0 for Bigger Datasets. Molecular and Biology Evolution 33:1870-1874.

Machado, E.M.S., R.M. Rodriguez-Jasso, J.A. Teixeira, and S.I. Mussatto. 2012. Growth of fungal strains on coffee industry residues with removal of polyphenolic compounds. Biochemical Engineering Journal 60:87-90.

Onwubuya, K., A. Cundy, M. Puschenreiter, J. Kumpiene, B. Bone, J. Greaves, et al. 2009. Developing decision support tools for the selection of "gentle" remediation approaches. Science of the Total Environment 407:61326142. 
Pérez, J., J. Muñoz-Dorado, T. de la Rubia, and J. Martínez. 2002. Biodegradation and biological treatments of cellulose, hemicellulose and lignin: an overview. Int. Microbiol. 5:53-63.

Ravanal, M.C., M. Alegría-Arcos, F.D. Gonzalez-Nilo, and J. Eyzaguirre. 2013. Penicillium purpurogenum produces two GH family 43 enzymes with $\beta$-xylosidase activity, one monofunctional and the other bifunctional: Biochemical and structural analyses explain the difference. Archives of Biochemistry and Biophysics 540:117-124.

Rubio-Senent, F., A. Lama-Munoz, G. Rodriguez-Gutierrez, and J. Fernandez-Bolanos. 2013. Isolation and identification of phenolic glucosides from thermally treated olive oil byproducts. Journal of Agricultural and Food Chemistry 61:1235-1248.

Sanchez, C. 2009. Lignocellulosic residues: Biodegradation and bioconversion by fungi. Biotechnology Advances 27:185-194.
Schouten, A., L. Wagemakers, F.L. Stefanato, R.M. van der Kaaij, and J.A. van Kan. 2002. Resveratrol acts as a natural profungicide and induces self-intoxication by a specific laccase. Molecular Microbiology 43:883-894.

Umsza-Guez, M.A., A.B. Díaz, I. de Ory, A. Blandino, E. Gomes, and I. Caro. 2011. Xylanase production by Aspergillus awamori under solid state fermentation conditions on tomato pomace. Brazilian Journal of Microbiology 42:1585-1597.

Valenzuela J.F., L. Pinuer, A.G. Cancino, and R.B. Yáñez. 2015. Effect of $\mathrm{pH}$ and dilution rate on specific production rate of extra cellular metabolites by Lactobacillus salivarius UCO_979C in continuous culture. Applied Microbiology Biotechnology 99:6417-6429. 